\title{
Un sistema de análisis espectral permite optimizar la iluminación de obras de arte y patrimonio cultural
}

\begin{abstract}
El sistema Matissse se presentaba el 23 de febrero de 2019 a la comunidad museística en el Museo Nacional Centro de Arte Reina Sofía durante la XIX Jornada de Conservación de Arte Contemporáneo. Se trata de un sistema de análisis espectral patentando, único en el mundo, que permite deducir la forma más óptima para iluminar una obra de arte. Uno de los factores más importantes del método Matisse respecto a otros sistemas de iluminación convencionales es que utiliza tecnología LED y elimina las radiaciones IR y UV conocidas por influir negativamente en la conservación de las obras de arte y patrimonio cultural.
\end{abstract}

Michel Silva Fino | Departamento de I+D+i, AS DE LED

Url de la contribución <www.iaph.es/revistaph/index.php/revistaph/article/view/4634>

Son conocidas las ventajas que aporta la tecnología LED prácticamente a la totalidad de sectores y actividades humanas. Ahorro energético, disminución de emisiones, versatilidad de diseño, prolongada vida útil de las luminarias, bajo mantenimiento, alta eficiencia, etcétera. Existe la falsa creencia de que el LED es fácil de comercializar, en consecuencia el mercado se encuentra saturado de muchos vendedores que carecen de los conocimientos necesarios en materias eléctricas, luminotécnicas y fotométricas. Al ser la iluminación de espacios culturales uno de los lugares más sensibles al mal criterio, la implantación de la tecnología LED ha avanzado lentamente en ese entorno. Actualmente, muchos museos emplean antiguas tecnologías para la iluminación artificial de sus instalaciones y obras; las más extendidas son el halogenuro metálico y el foco halógeno, cuya fabricación está prohibida desde junio del presente año.

En 2004, la Commission Internationale de l'Eclairage (CIE) publicó el informe técnico 157:2004 Control del daño por radiación óptica a objetos de museo, en el que se establecen los protocolos de iluminación de obras artísticas, que se definen desde un equilibrio entre los problemas derivados por la acción fotoquímica de la radiación y los problemas de fidelidad cromática de la visualización obra. Esta norma, ampliamente aceptada, no tiene en cuenta la rápida incorporación de los iluminantes de estado sólido (light emitting diode, LED). La introducción de este tipo de iluminación presenta grandes ventajas. Entre otras, la restricción al rango visible del espectro de radiación óptica del LED evita no solo los problemas derivados de las colas de rango ultravioleta (UV) e infrarrojo (IR) que otros iluminantes tenían -como los basados en lámparas de incandescencia o halogenuros-, sino que favorece los procesos de medición óptica, al poder utilizar fotometría convencional y no sistemas de medición radiométrica. Partiendo de esta y otras publicaciones, se han caracterizado una serie de tablas que son ampliamente conocidas por conservadores y comisarios en general. Por citar un ejemplo, tenemos la de valores acumulativos de radiación luminosa (ver tabla de debajo).

No hay publicaciones que expongan métodos que incluyan factores como el espectro de la fuente de luz, la evaluación de la paleta de colores de la obra para optimizar la iluminación, la curva de respuesta espectral fotópica humana, etcétera.

\begin{tabular}{lll} 
Grupo & Materiales & Valores $(\mathbf{l x} \cdot \mathbf{h} / \mathbf{a n ̃ o})$ \\
\hline A & $\begin{array}{l}\text { Acuarelas, telas, papel, graba- } \\
\text { dos, etc. }\end{array}$ & 50.000 \\
& & \\
B & Óleos, témperas, hueso, marfil, & 600.000 \\
& cuero, etc. & \\
& & \\
C & Piedra, metal, cerámica, fotos \\
& en blanco y negro
\end{tabular}

Valores acumulativos de exposición máximos recomendados 
Cabe mencionar también que el deterioro en las obras de arte está causado no por la luz que refleja, sino por la que absorbe. Si una obra de arte posee una paleta de colores muy oscura, estará absorbiendo la mayoría de la luz, y reflejando una cantidad mínima. Eso obliga también a emplear unos valores de iluminancia más elevados o salas muy oscuras para poder exponerla correctamente. Esto es así porque hasta ahora no se disponía de un método que pudiera analizar la obra de arte de forma individual, y que esté orientado a disminuir la emisión de luz que absorberá la obra, y emplear solo los componentes longitudinales que reflejará que, a fin de cuentas, son los colores que verá el ojo humano.

El Sistema Matisse fue presentado a la comunidad museística en la 19. ${ }^{\circ}$ Jornada de Conservación de Arte Moderno en el Museo Nacional Centro de Arte Reina Sofia, en una ponencia preparada conjuntamente por la empresa LED Y SPA, el Departamento de Restauración del Instituto Valenciano de Arte Moderno, y la Facultad de Óptica de la Universidad de Valencia.

\section{Funcionamiento del método}

Utilizamos una luminaria LED que ofrece como principal característica su capacidad para variar los parámetros de la luz: espectro, la temperatura de color, el índice de reproducción cromática, las coordenadas cromáticas en el espacio de color, etcétera. La luminaria se controla desde un ordenador portátil, que posee un software desarrollado especialmente para el proceso y un bus de comunicación que permite programar perfiles de luz y hacer que los reproduzca con elevada precisión. Adicionalmente, el equipo posee un sistema óptico que nos facilita controlar el ángulo y la forma del haz de luz, permitiendo adaptar la metodología del ensayo a obras de distintas formas, dimensiones, y proporciones. Los equipos de espectrocolorimetría se disponen a la distancia adecuada de la obra, que en adelante llamaremos objeto bajo estudio (OBE), para que la sonda subtienda el ángulo de medida adecuado para la muestra, con el propósito de leer la luz reflejada por el OBE, que llega desde la luminaria LED. Para el desarrollo del protocolo se determinarán los siguientes parámetros: temperatura de color, coordenadas cromáticas en los espacios del color CIE1931 y CIE1976, índice de reproducción cromática (CRI), pureza, iluminancia, longitud de onda dominante, flicker y distribución espectral.

La premisa básica del Método Matisse es la comparación entre la luz emitida y la luz reflejada; una vez determinadas, se puede calcular cuál es el espectro de absorción promedio del OBE. Dado que las emisiones IR

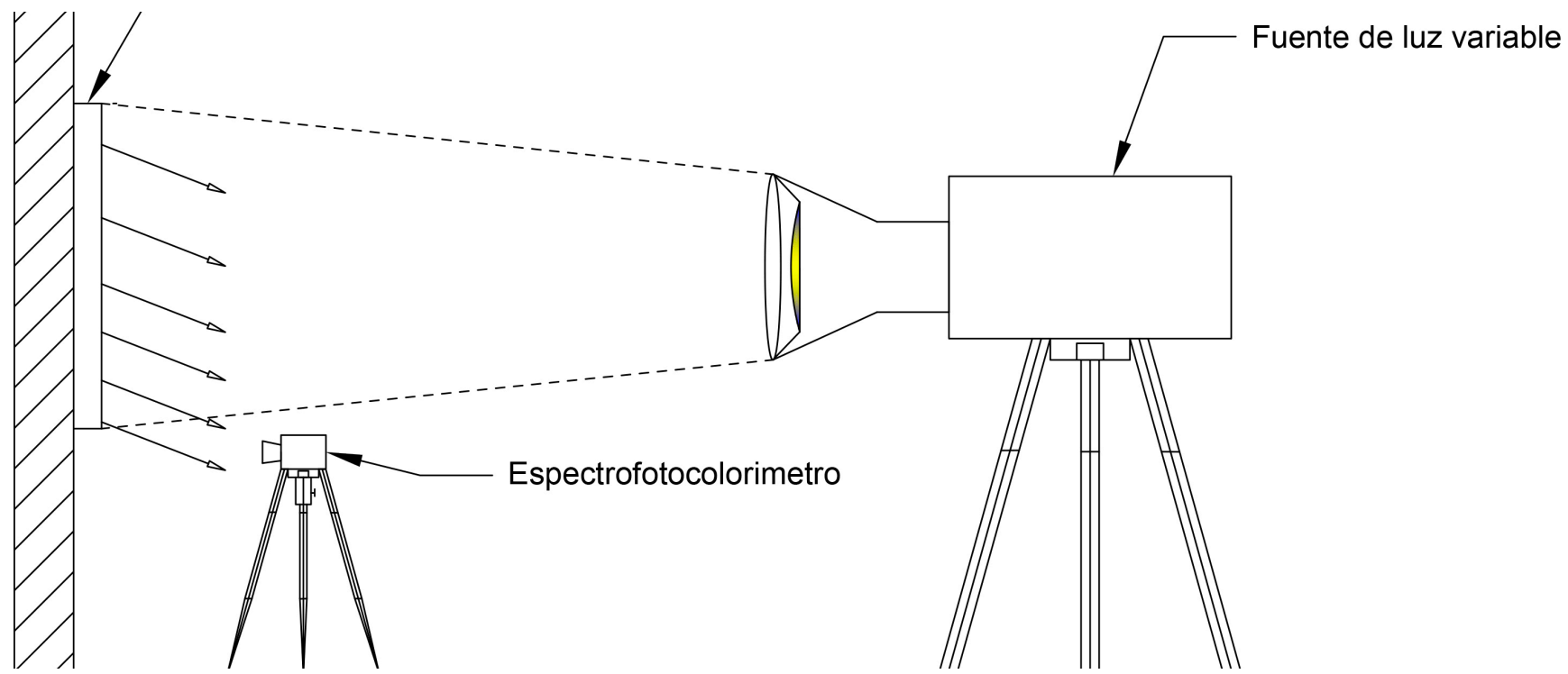

Esquema de montaje del instrumental frente al OBE durante la etapa de ensayo | fuente propia 


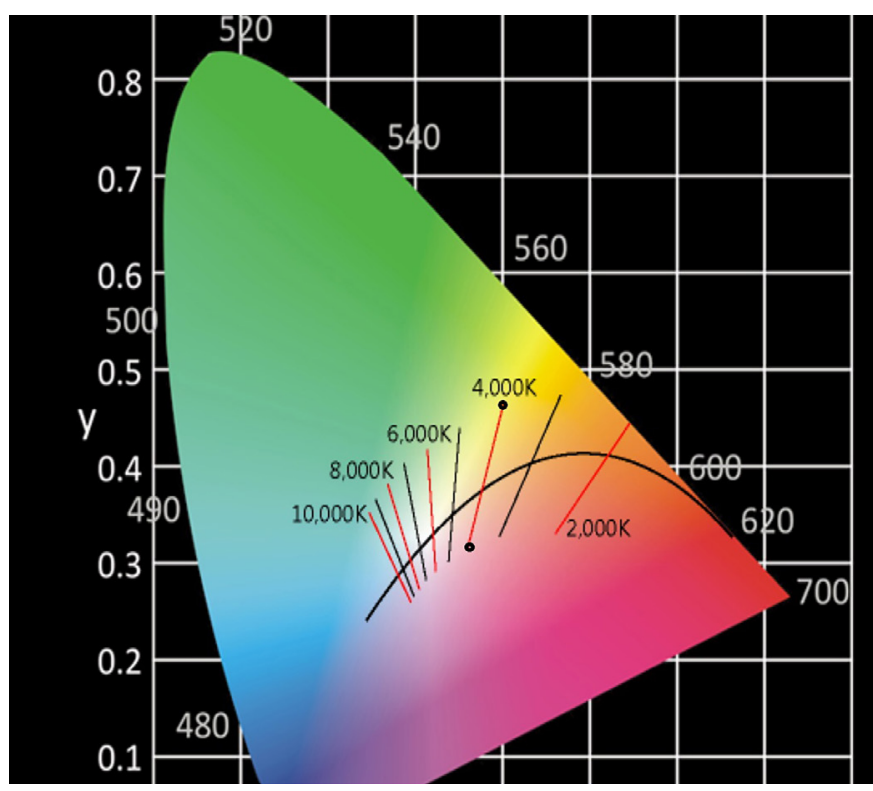

Gráfico de espacio del color CIE 1931 con coordenadas marcadas en ambos extremos de los 4000K | fuente propia

y UV del LED son despreciables, se utilizará tan solo el rango visible del espectro, considerado desde los 380nm a los 780nm en coincidencia tanto con la curva de sensibilidad fotópica del observador patrón propuesta por la CIE (1924) como con el rango de emisiones de la tecnología LED. En primer lugar, se optimizará la iluminación en función de la temperatura de color del LED, para lo que se realiza un barrido entre los $2300 \mathrm{~K}$ y los $8000 \mathrm{~K}$. En este barrido se determina la temperatura de color que mantiene más inalterado el blanco de referencia en el diagrama cromático CIE XY, determinando asimismo cuál es la temperatura de color que más luz refleja el $\mathrm{OBE}$, que se considerará como temperatura de iluminación. En un segundo paso de optimización se mejora la reproductibilidad cromática a través de una biblioteca de perfiles de iluminación que reproduzcan la temperatura de color obtenida

En la figura de arriba se puede apreciar cómo obtener perfiles de luz dentro de la misma temperatura de color $4000 \mathrm{~K}$, donde en un extremo tenemos tintes amarillos/ verdes, y en el otro extremo tendremos tintes rosas/azules. A pesar de ser la misma temperatura de color, las diferencias de los dos perfiles de luz resultan muy evidentes. A simple vista se aprecian sus notables diferen- cias cromáticas, en consecuencia, su efecto sobre un elemento iluminado también son objeto de consideración. Con este procedimiento se evalúa la reflectividad de cada perfil de luz medido a lo largo de la misma temperatura de color hasta obtener un punto en el que se dé la mejor respuesta posible. Ese punto, que resulta ser un perfil de luz optimizado para la conservación del OBE, se tomará como parte central de un último proceso de mediciones, que se efectúan haciendo pequeños cambios en los parámetros de la luminaria variable para hacer que la luz tenga tendencias hacia distintas direcciones en el espacio del color a partir de esa medición central. Cada vez que se proyecta un perfil de luz sobre el OBE se toman registros con los equipos de espectrofotocolorimetría para su posterior análisis

El resultado del proceso será una referencia o características generales de la fuente de luz que representará fielmente los colores del OBE teniendo en cuenta las particularidades de la visión humana y favorecerá la conservación preventiva de la obra al disminuir la luz que se proyecta de forma innecesaria, siendo toda esta información útil para el diseño y fabricación de una luminaria a medida. 